\title{
Two Dimensional Harmonics Retrieval in Zero-Mean Independent Multiplicative and Additive Noises
}

\author{
Zheng- Wei ZHANG ${ }^{1, a}$, Zhong CHEN ${ }^{1}$, Li-Hui WANG ${ }^{2}$ \\ 1 The $28^{\text {th }}$ Research Institute, China Electronics Technology Group Corporation, Nanjing, China \\ 2 Southeast University, School of instrument science and engineering, Key laboratory of micro-inertial instrument and advanced navigation \\ technology, Ministry of education, Nanjing, China
}

\begin{abstract}
For some theoretical problems and computational inefficiency, the problem of estimating the frequencies of two dimensional harmonics in zero-mean independent multiplicative and additive noises is not resolved completely. Existing methods based on cyclic statistics assume that the noise is non-Gaussian, and that the shape of the noise's distribution is not symmetrical. A novel approach which imposes no constraints on the distribution or the color of noises is developed. It relies on fourth-order special cumulant or moment slices. The fourth-order moment spectrum is shown to have computational advantages over existing method. As a byproduct, the variance of the noises can also be estimated directly from the spectrum. Finally we give as an illustrative example a complete experiment to corroborate the theoretical results. The results of this experiment do show preliminarily that the new method is valid and efficient for the problem of harmonics retrieval in zero-mean independent multiplicative and additive noises.
\end{abstract}

\section{Introduction}

Estimating harmonics with multiplicative noises using higher order spectra or cyclic statistics has been drawing a great deal of attention. Dwyer [1] estimated the frequencies of one-dimensional harmonic in the presence of Gaussian multiplicative and additive noises based on the spectrum of a special fourth-order cumulant. Zhou [2] developed a discrete time-averaged moment framework for retrieval of one dimensional harmonics with multiplicative noise using the notion of Fourier series moment polyspectra. And in contrast to what assumed in [1], both the additive and the multiplicative noises need not be Gaussian, as long as they are mutually independent with absolutely summable cumulants. Giannakis [3] studied the retrieval problem of one dimensional harmonics using cyclic statistics, and proved that as far as frequency estimation is concerned, cyclic and higher order statistics based approaches are equivalent. Kareem [4] provided a measure of the multiplicative nonlinear interactions of frequency components and offers physical insight into the nature and characteristics of nonlinear fluctuations Baars [5] reduced essentially to spectral Linear Stochastic Estimation when only first-order terms are considered, and is therefore presented in the context of stochastic estimation as spectral Higher-Order Stochastic Estimation. All these methods assume different noise background. For details, please refer these references and therein.

Frequencies estimation of two dimensional harmonics is the main problem [6-7] at present. Some methods above and others assume that the phase of harmonics is a uniformly distributed or Gaussian process, and that the mean of the noises is nonzero. However, the phase is always deterministic, as a single record of data is often available in practice, and the means of noises are not necessarily nonzero. Actually, correlativity between noises is also not guaranteed. Here, a novel method to estimate harmonics in zero-mean independent multiplicative and additive noises is developed based on higher order spectra using only a single record of data. This method only requires that the noises are mixing and stationary, and imposes no constraints on the distribution or color of the noises.

\section{Base Problem Statements}

The complex two dimensional harmonics model studied widely takes in discrete time the form

$$
x(m, n)=\sum_{l=1}^{L} s_{l}(m, n) e^{-j\left(m \omega_{l 1}+n \omega_{l 2}+\phi_{l}\right)}+\eta(m, n),
$$

\footnotetext{
a Corresponding author: ytzzw126@126.com
} 
where $m=0,1, \cdots T_{1}-1, n=0,1, \cdots T_{2}-1, L$ represents the number of signal, $\left(\omega_{l 1}, \omega_{l 2}\right)$ denotes the $l$ th frequency pair, both $\omega_{l 1}$ 's and $\omega_{l 2}$ 's are distinct and nonzero mod ( $2 \pi), \phi_{l}$ represents the $l$ th phase, $s_{l}(m, n)$ represents the $l$ th multiplicative noise, $\eta(m, n)$ represents the additive noise.

Definition 1 Let the $(u+v)$ th order moment of the complex harmonic $x(m, n)$ with $u$ unconjugated and $v$ conjugated entries be

$$
\begin{aligned}
m_{u v x}(m, n, \boldsymbol{\tau}, \boldsymbol{\xi}) \triangleq E & \left\{x(m, n) x\left(m+\tau_{1}, n+\xi_{1}\right) \cdots x\left(m+\tau_{u-1}, n+\xi_{u-1}\right)\right. \\
& \left.\times x^{*}\left(m+\tau_{u}, n+\xi_{u}\right) \cdots x^{*}\left(m+\tau_{u+v-1}, n+\xi_{u+v-1}\right)\right\},
\end{aligned}
$$

where $\boldsymbol{\tau}=\left\{\tau_{1}, \cdots, \tau_{u+v-1}\right\}, \boldsymbol{\xi}=\left\{\xi_{1}, \cdots, \xi_{u+v-1}\right\}$, the symbol '* , denotes conjugate operator.

Similar notational convention will be adopted for the $(u+v)$ th order cumulant $c_{u v x}(m, n ; \boldsymbol{\tau}, \boldsymbol{\xi})$. Generally speaking, absolute cumulant summability does not imply absolute moment summability unless $x(m, n)$ is zero-mean and $u+v=1,2,3$, in which case cumulant coincide with moments. For zero-mean Gaussian process, the odd order moments equal 0 , but not for even order. However, all the cumulants with third-order or higher equal 0 . So higher order statistics is often applied to suppress additive Gaussian noises.

In general, all the noises in Eq. (1) are assumed to satisfy the mixing condition [8] as follows, $\forall u, v \geq 0, u+v=1, \cdots, 8$

$$
\sum_{\tau_{1} \cdots \tau_{u+v-1}=-\infty}^{\infty} \sum_{\xi_{1} \cdots \xi_{u+v-1}=-\infty}^{\infty}\left|c_{u v s}(\boldsymbol{\tau}, \boldsymbol{\xi})\right|<\infty
$$

The mixing condition holds similarly for $\eta(m, n)$ and $x(m, n)$.

If $\phi_{l}$ 's in Eq. (1) are uniformly i.i.d. random variables in $(-\pi, \pi]$ and independent of $s_{l}(m, n)$ as well as $\eta(m, n)$, and $s_{l}(m, n)$ and $\eta(m, n)$ are stationary, we can easily prove that $x(m, n)$ is stationary but not always mixing. We assert that under the deterministic $\phi_{l}$ assumption, $x(m, n)$ is nonstationary but mixing [9]. Now we give the assumptions in Eq. (1)

AS1. $s_{l}(m, n)$ 's and $\eta(m, n)$ are zero-mean, stationary, mixing, real and mutually independent.

AS2. The $\phi_{l}$ 's are deterministic.

The uniform phase randomization assumption prevents $x(m, n)$ from being Gaussian, which otherwise would render Dwyer's fourth-order cumulant statistics method [1] useless for frequency estimation. However, for a single record of data, $\phi_{l}$ is always deterministic, so $x(m, n)$ is Gaussian under the assumption in Dwyer's method that $s_{l}(m, n)$ and $\eta(m, n)$ are both Gaussian and mutually independent. It is necessary to define special cumulant in order to prevent frequency information contained in the cumulant from losing. Dou's method [6] fails since it assumes that the noises are mutually correlative. Wang's method [7] fails for its constrain imposed on the distribution of the noises.

For the assumptions above, A novel time averaged moment approach is established in the next section, where noises $s_{l}(m, n)$ 's and $\eta(m, n)$ are allowed to be nonGaussian, provided that they satisfy the mixing condition, Eq. (3), which can be met in practical setups.

\section{Harmonics Retrieval Algorithm}

Let us rewrite Eq. (1) as

$$
x(m, n)=\sum_{l=1}^{L+1} S_{l}(m, n) e^{-j\left(m \omega_{l 1}+n \omega_{l 2}\right)},
$$

where $\quad S_{l}(m, n)=s_{l}(m, n) e^{j \phi_{l}} \quad, \quad S_{L+1}(m, n) \triangleq \eta(m, n)$, $\left(\omega_{(L+1) 1}, \omega_{(L+1) 2}\right) \triangleq(0,0), \phi_{L+1} \triangleq 0$. Thus we consider additive disturbance as a special multiplicative noise.

Let $\boldsymbol{\tau}=(0, \tau, \tau), \boldsymbol{\xi}=(0, \xi, \xi)$, we get the special fourth-order moment of $x(m, n)$ according to Eq. (2) as follows

$$
m_{22 x}(m, n, \boldsymbol{\tau}, \boldsymbol{\xi}) \triangleq E\left\{x(m, n) x(m, n) x^{*}(m+\tau, n+\xi) x^{*}(m+\tau, n+\xi)\right\}
$$

Since $x(m, n)$ is a zero-mean stationary process, we get its special fourth-order cumulant according to the M-C formula

$c_{22 x}(m, n, \boldsymbol{\tau}, \boldsymbol{\xi})=m_{22 x}(m, n, \boldsymbol{\tau}, \boldsymbol{\xi})-2 m_{11 x}{ }^{2}(m, n, \tau, \boldsymbol{\xi})-m_{20 x}{ }^{2}(m, n, 0,0)$

Definition 2 Define the special $(2+2)$ th order time averaged moment of $x(m, n)$ be

$\bar{m}_{22 x}(\boldsymbol{\tau}, \boldsymbol{\xi}) \triangleq \lim _{T_{1} \rightarrow \infty} \lim _{T_{2} \rightarrow \infty} \frac{1}{T_{1} T_{2}} \sum_{m=0}^{T_{1}-1} \sum_{n=0}^{T_{2}-1} m_{22 x}(m, n, \boldsymbol{\tau}, \boldsymbol{\xi}),($

where $m_{22 x}(m, n, \boldsymbol{\tau}, \boldsymbol{\xi})$ is given by Eq. (5). 
Definition 3 The special $(2+2)$ th order time averaged moment spectrum of $x(m, n)$ corresponding to Definition 2 is

$$
\begin{aligned}
& \bar{M}_{22 x}(\alpha, \beta) \triangleq \lim _{T_{1} \rightarrow \infty} \lim _{T_{2} \rightarrow \infty} \frac{1}{T_{1} T_{2}} \sum_{\tau=0}^{T_{1}-1} \sum_{\xi=0}^{T_{2}-1} \bar{m}_{22 x}(\tau, \xi) e^{-j \alpha \tau} e^{-j \beta \xi} \\
& =\lim _{T_{1} \rightarrow \infty} \lim _{T_{2} \rightarrow \infty} \frac{1}{T_{1}^{2} T_{2}^{2}} \sum_{\tau=0}^{T_{i}-1} \sum_{\xi=0}^{T_{2}-1} \sum_{m=0} \sum_{n=0}^{T_{1}-1} T_{T_{2}-1} E\left\{x^{2}(m, n)\left(x^{*}(m+\tau, n+\xi)\right)^{2}\right\}
\end{aligned}
$$

An important identity used in below derivation is

$\lim _{T \rightarrow \infty} \frac{1}{T} \sum_{t=0}^{T-1} e^{j \omega t}=\delta(\omega) \triangleq \begin{cases}1 & \alpha=0 \bmod (2 \pi) \\ 0 & \alpha=\text { others }\end{cases}$

Now we derive the fourth-order time averaged moment approach. Let

$$
\begin{aligned}
& x^{2}(m, n)=\sum_{l=1}^{L+1} S_{l}^{2}(m, n) e^{-2 j\left(m \omega_{11}+n \omega_{l 2}\right)} \\
& +2 \sum_{\substack{\left.l_{1}=1, l_{l}=1 \\
l_{1} \neq l_{2}\right)}}^{\mathrm{L}+1} S_{l_{1}}(m, n) S_{l_{2}}(m, n) e^{-j m\left(\omega_{l_{11}}+\omega_{l_{2} 1}\right)} e^{-j n\left(\omega_{l_{12}}+\omega_{l_{2}}\right)} \\
& \left(x^{*}(m+\tau, n+\xi)\right)^{2}=\sum_{l=1}^{L+1}\left(S_{l}^{*}(m+\tau, n+\xi)\right)^{2} e^{2 j\left((m+\tau) \omega_{n}+(n+\xi) \omega_{l 2}\right)} \\
& +2 \sum_{\substack{l_{1}=1, l_{2}=1 \\
\left(l_{1} \neq L_{2}\right)}}^{\mathrm{L}+1} S_{l_{1}}{ }^{*}(m+\tau, n+\xi) S_{l_{2}}{ }^{*}(m+\tau, n+\xi) e^{j(m+\tau)\left(\omega_{n_{11}}+\omega_{l_{2}}\right)} \\
& * e^{j(n+\xi)\left(\omega_{12}+\omega_{12}\right)}
\end{aligned}
$$

Substituting Eq. (9) and (10) into Eq. (7), we obtain

$\bar{M}_{22 x}(\alpha, \beta)=\sum_{l=1}^{L}\left|m_{20 S_{l}}(\boldsymbol{\theta}, \boldsymbol{\theta})\right|^{2} \delta\left(a-2 \omega_{l 1}\right) \delta\left(\beta-2 \omega_{l 2}\right)+\left|m_{20 \eta}(\boldsymbol{\theta}, \boldsymbol{\theta})\right|^{2} \delta(0) \delta(0)$.

From Eq. (12), we know that $\bar{M}_{22 x}(\alpha, \beta)$ peaks at $\left(2 \omega_{11}, 2 \omega_{12}\right), \cdots,\left(2 \omega_{(L+1) 1}, 2 \omega_{(L+1) 2}\right)$, which prompts the following harmonics retrieval expression

$$
\left\{\left(\omega_{l 1}, \omega_{l 2}\right)\right\}_{l=1}^{L+1}=\frac{1}{2} \underset{\left(\alpha_{1}, \beta_{1}\right), \cdots,\left(\alpha_{L+1}, \beta_{L+1}\right)}{\arg \max } \sum_{l=1}^{L+1}\left|\hat{\bar{M}}_{22 x}\left(\alpha_{l}, \beta_{l}\right)\right|^{2}
$$

Actually, we also estimate the variance of the noises, since

$$
\begin{aligned}
\left|m_{20 s}(0,0)\right|^{2} & =\mid E_{s}\left\{\left.(x(m, n) x(m, n)\}\right|^{2}\right. \\
& =\left|\mathrm{D}_{\mathrm{s}}(\mathrm{x}(\mathrm{m}, \mathrm{n}))+\mathrm{E}_{\mathrm{s}}^{2}(\mathrm{x}(\mathrm{m}, \mathrm{n}))\right|^{2}=\left|\mathrm{D}_{\mathrm{s}}(\mathrm{x}(\mathrm{m}, \mathrm{n}))\right|^{2}=\sigma_{s}^{4}
\end{aligned}
$$

\section{Performance Analysis}

Before introducing the sample estimator, we wish to show an interesting equivalence

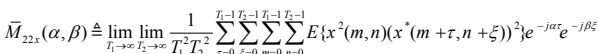

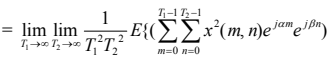

$$
\begin{aligned}
& \left.\left.\times \sum_{\tau=0}^{T_{1}-1 \sum_{\xi=0}-1}\left(x^{*}(m+\tau, n+\xi)\right)^{2} e^{-j(m+r)} e^{-j \beta(n+\xi)}\right)\right\}
\end{aligned}
$$

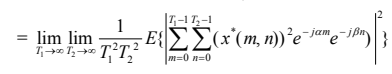

$$
\begin{aligned}
& =\lim _{T_{1} \rightarrow \infty I_{2} \rightarrow \infty} \frac{1}{T_{1}^{2} T_{2}^{2}} E\left\{\left|D F T\left(\left(x^{*}(m, n)\right)^{2}\right)\right|^{2}\right\}
\end{aligned}
$$

From Eq. (15), the natural estimator for $\bar{M}_{22 x}(\alpha, \beta)$ is

$$
\widehat{\bar{M}}_{22 x}(\alpha, \beta)=\frac{1}{T_{1}^{2} T_{2}^{2}}\left|\operatorname{DFT}\left(\left(x^{*}(m, n)\right)^{2}\right)\right|^{2}
$$

Comparing Eq. (15) with Eq. (14), we know that $\overline{\bar{M}}_{22 x}(\alpha, \beta)$ is asymptotically unbiased and can be computed efficiently by using FFT.

For similar consistency proof, we refer readers to the literature [2, 10] and therein. In literature [2] we also see that the variance of the estimator has a fast converging rate of $O\left(T^{-3}\right)$.

\section{Simulation Results}

Since noises in Eq. (1) satisfy AS1 and AS2 different from that in literature [6], the methods there are apparently invalid. The method in literature [7] is also designed specially for harmonics retrieval problem in zero-mean independent noises, whereas it requires the multiplicative noises in Eq. (1) are nonsymmetrical or non-Gaussian. Here we only give an example to show our method is valid for the special conditions, despite that our method does not need to impose any constrains on the distribution or color of all the noises in Eq. (1).

Suppose there are three components in model (1) with parameters $\left(\omega_{11}, \omega_{12}\right)=(0.50,0.75),\left(\omega_{21}, \omega_{22}\right)=(1.15,2.25)$, $\left(\omega_{31}, \omega_{32}\right)=(2.50,1.50), \phi_{1}=0.2, \phi_{2}=0.1, \phi_{3}=0.4$, respectively. The noises are produced by second order MA model driven by different Gaussian white random process as follows

$$
\begin{aligned}
& s_{1}(m, n)=e_{1}(m, n)-0.5 e_{1}(m-1, n)-0.3 e_{1}(m-2, n) \\
& s_{2}(m, n)=e_{2}(m, n)-0.3 e_{2}(m-1, n)-0.7 e_{2}(m-2, n) \\
& s_{3}(m, n)=e_{3}(m, n)-0.35 e_{3}(m-1, n)-0.65 e_{4}(m-2, n) \\
& \eta(m, n)=e_{4}(m, n)-0.4 e_{4}(m-1, n)-0.6 e_{4}(m-2, n)
\end{aligned}
$$

where $e_{1}(m, n), e_{2}(m, n), e_{3}(m, n)$, and $e_{4}(m, n)$ are Gaussian random processes with mean zero and variance 1 . The data length is $1024 \times 1024$.

Figure 1 and Figure 2 show the estimation results for $\hat{\bar{M}}_{22 x}(\alpha, \beta)$ using the method proposed in literature [6] and [7], respectively. Figure 3 and Figure 4 are the results by the method in this paper, where $\bar{M}_{22 x}(\alpha, \beta)$ precisely peaks 
at $\left(\omega_{11}, \omega_{12}\right)=(1.00,1.50), \quad\left(\omega_{21}, \omega_{22}\right)=(2.30,4.50) \quad$ and $\left(\omega_{31}, \omega_{32}\right)=(5.00,3.00)$

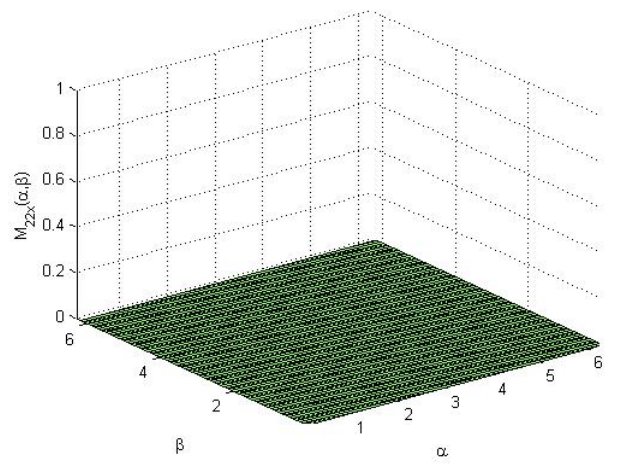

Figure 1. Results by Method in Ref. [6]

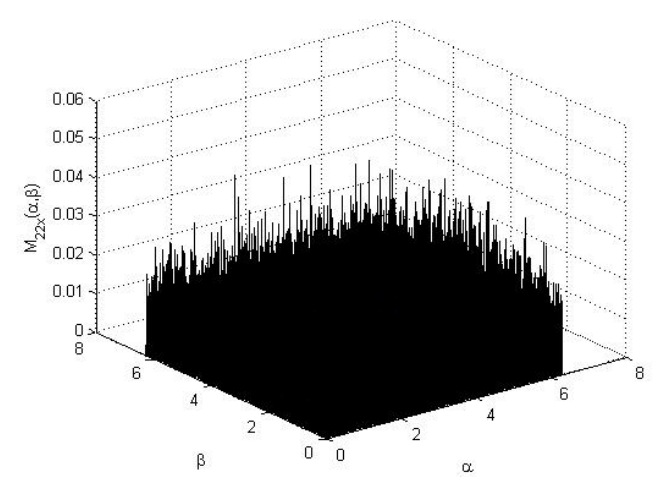

Figure 2. Results by Method in Ref. [7]

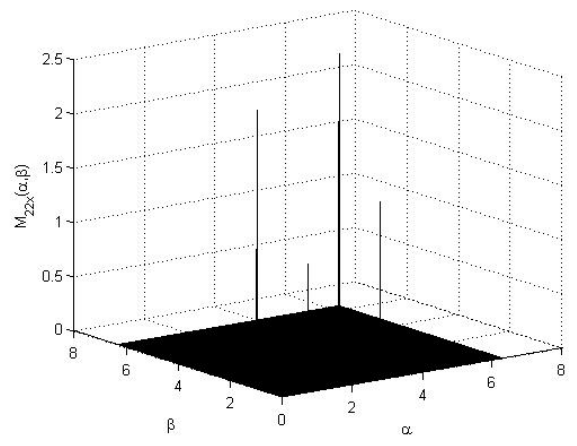

Figure 3. Results by Method in This Paper

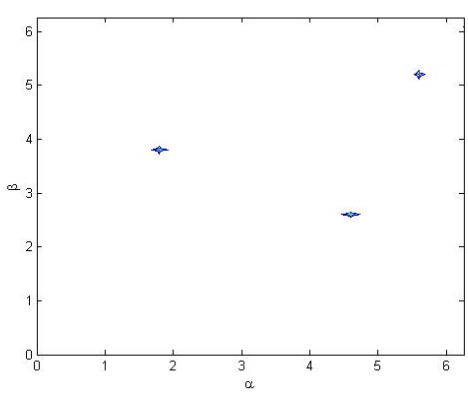

Figure 4. The Contour of $\overline{\bar{M}}_{22 x}(\alpha, \beta)$

As analyzed above, the spectrum peak at $(2 \pi, 2 \pi)$ in Fig. 3 is caused by the additive noise. The frequencies and the variances of the noises estimated from Eq. (21) and (22) are shown in Table 1. Increasing the data length, we can get more precise estimation, since the estimator is asymptotically unbiased.

Table 1. Estimation Results

\begin{tabular}{|l|l|l|l|}
\hline$l$ & $\omega_{l 1}$ & $\omega_{l 2}$ & $\delta_{s l}^{2}$ \\
\hline 1 & 0.5000 & 0.7485 & 0.8736 \\
\hline 2 & 1.1504 & 2.2488 & 1.2032 \\
\hline 3 & 2.5003 & 1.5002 & 1.6230 \\
\hline 4 & 0 & 0 & 2.1012 \\
\hline
\end{tabular}

\section{Conclusions}

We proposed a harmonics retrieval method based on a special time averaged moment and cumulant for the situations where the multiplicative and additive noises are zero-mean independent stationary processes. Here we consider the deterministic phases since we always obtain a single record data in practice, and only require the noises are mixing. The method in this paper does not need to impose any constraints on the distribution and color of the noises, and the estimator can be computed efficiently by using FFT. As a by product, the variances of the noises which appear as the amplitudes of the harmonics peaks of the special time averaged moment spectrum slice can also be estimated easily. Experiments confirm to the correctness and validity of the method.

But as mentioned in literature [2], the phenomenon that the frequency pairs are restricted to $(0, \pi]$ in order to avoid aliasing also happened in this paper. To develop a method without these limits will be the further research direction.

\section{References}

1. R. R. Dwyer., J. Acoust. Soc. Amer., 90, 918(1991) 
2. G. T. Zhou, G. B. Giannakis., IEEE. J. Oceanic Eng., 19, 529(1994)

3. G. B. Giannakis, G. T. Zhou., IEEE Trans. Signal Processing, 43, 2217(1995)

4. A Kareem, Y Li. Probabilistic Methods in Civil Engineering, 384(2015)

5. WJ Baars, CE Tinney., Physics of Fluids, 8, 2182(2014)

6. H. J. Dou, S. X. Wang, F. Wang., Acta Electronica Sinica, 32, 83(2004)

7. F. Wang, S. X. Wang, H. J. Dou., Acta Electronica Sinica, 31, 1522 (2003)

8. G.T. Zhou, Random amplitude and polynomial phase modeling of nonstationary process using higher order and cyclic statistics., Canada Charlottesville, University of Virginia, VA 22903-2442

9. Z.W. Zhang, Y.Y. Fan, Z. Li., Journal of Information and Computational Science, 4, 387(2007)

10. H.W. Li, Q.S. Cheng, B. Z., Signal processing proceedings, Fourth ICSP'98, 43(1998) 